\title{
A Robust Mechanism for Robot Team Formation
}

\author{
Jing Wu, Yi Jiang*, Kairong Li, Zhou Xu \\ School of Information Engineering, Yangzhou University, Yangzhou 225127, China \\ *Corresponding Author: Jiangyi@yzu.edu.cn
}

\begin{abstract}
With the development of AI, the job of robots became more complex and diversified, due to the limited capacity of a single robot, multiple robots cooperation is used to accomplish complex tasks. Based on the problem of how to form a cooperative team, in this paper we proposed a robust mechanism for robot team formation (RMTF). First the mechanism designed a SC algorithm for select team coordinator, and then used the CRN algorithm to check teams who can complete the task. Finally, the OT algorithm is used to optimize the team to perform the task. In this paper, an example is given to illustrate the robustness of the mechanism, compared with the existing mechanism of robot team formation, RMTF increased the team's robustness, and ensured the stability of the team to complete the task.
\end{abstract}

Keywords: robustness, team formation, algorithm, mechanism

\section{Introduction}

Task oriented team formation is the best way to solve the task, and team formation is associated with Multiple Robot System(MRS).With the rapid development of computer technology and artificial intelligence, robot technology is changing with each passing day. The work requirements for robot change from simple service to complex task, Multiple robot team has the ability to handle much stronger than the individual robot, not only can finish the task of individual robot can complete, but also the task that individual robot can' t complete. With the cooperation of multiple robots, the complicated task can be divided into a number of simple sub task, we can effectively complete the task by using multiple robot teams.

The main idea of this paper is multi robot cooperation that each robot has several kinds of abilities, according to different abilities of each robot, multi robot cooperate to complete the task. To formation a team satisfied all the technical requirements of the task, and the cost is not greater than the budget of the task. And in here we adopt centralized-decentralized programming which has a coordinator to accomplish internal coordinate work in each team. For example, the team cannot complete the task because of a robot break down, the coordinator can coordinate the other robot who has the same skill to complete the job instead.

Here is the structure of the article, introduces the related work of robot team formation in the second section. And the third section describes the model of robot team formation problem and the related definition, the fourth section introduces the negotiation mechanism between the task coordinator and robots. The fifth section of the paper is an example to illustrate the robustness of the mechanism, and the sixth section is the conclusion of the paper.

\section{Related Work}

Lots of works have been devoted to the problem of team formation. Team formation based on negotiation have been researched in paper[1], there is a team coordinator to negotiate with agents to join the team, and find other agents by neighbors to reduce communication cost. Nair et al.[2] committed to the formation of the team with the maximum expected value. Marcolino et al. [3] focused on the relationship between the diversity of a team and the strength of members to select a diversity team. Bachrach et al. [4] introduced the coalitional skill games, propose is to make a coalition among agents so that it can meet team efficiency.

Multi-Robot System have been studied in[5], aimed at form a task oriented robust team. Vidal et al. [6] focused on the research domain of task oriented, and shows the benefits of cooperation and selflessness. A distributed algorithm for the reconstruction of robot team is described in [7], take advantage of the function of robot local computing to determine whether a team needs to split or two teams need to reconfigure the process of 
merging. Kaminka et al.[8] introduced a kind of behavior based team architecture. Liemhetcharat et al. [9] studied the composed of configurable robots, and considered how to form a robust multi robot team.

\section{Model}

The cooperation between robots is an efficient and mutually beneficial manner to complete tasks. Robots work together can complete complicated tasks in parallel, which could not achieve by a single robot. But how the robots team formed?

\subsection{Model Description}

Task: Task $\mathrm{T}$ is defined by a 3-tuple $\langle S p, B u d, R e\rangle, S p$ is the initiator of $\mathrm{T}$, and $\mathrm{Bud}$ is the budget of task cost which is set by the initiator $\mathrm{Sp}, R e$ is the set of abilities that the task $\mathrm{T}$ request and $R e=\left\{\right.$ ability $_{m}$, ability $\left._{n}, \ldots\right\}$. Here we assume each task have and only have one initiator $\mathrm{Sp}$, and $\mathrm{Sp}$ would not contribute any ability to $\mathrm{T}$.

Robot: $\mathrm{R}=\left\{r_{1}, r_{2}, \ldots r_{m}\right\}$ is a set of robot who own different abilities, robot $r_{i}$ can be defined by a 2-tuple $\left\langle A_{r_{i}}, A C_{r_{i}}\right\rangle, \quad \mathrm{A}_{\mathrm{r}_{\mathrm{i}}}$ are the abilities that robot $\mathrm{r}_{\mathrm{i}} \in \mathrm{R}$ own, and each robot may own at least one ability. For instance, a 3-tuple $\left\langle\right.$ ability $\left._{i}, \operatorname{cost}_{i}, x\right\rangle$ denotes the cost $\operatorname{cost}_{i}$ and proficiency $\mathrm{x}$ to ability $_{i}$ of robot.

Selfish: Robot tends to join the team which can achieve more benefit is the selfish nature of robot. Assume a robot $r_{i}$ own two abilities ability $_{1}$ and ability $_{2}$, if task $T_{1}$ pay cost $_{1}$ to $r_{i}$ for ability $_{1}$, task $T_{2}$ pay cost $_{2}$ to $r_{i}$ for ability $_{2}$ and $\operatorname{cost}_{1}>\operatorname{cost}_{2}$, due to the selfish nature robot $r_{i}$ tend to join the team to implement task $T_{1}$.

\subsection{Model Definition}

Definition 1. (Team formation) A team formation problem can be described as a 3-tuple $\mathrm{TF}=\{\mathrm{TM}, \mathrm{TA}, \mathrm{US}$,$\} , where$ $\mathrm{TM}=\left\{\mathrm{r}_{1}, \mathrm{r}_{2}, \ldots \mathrm{r}_{\mathrm{k}}\right\}$ is the set of robots in the team, $\mathrm{TA}=\left\{\right.$ ability $_{1}$, ability $_{2}, \ldots$, ability $\left._{\mathrm{i}}\right\}$ is the set of abilities that the team members own, US $=\left\{\right.$ ability $_{\mathrm{p}}$, ability $\left._{\mathrm{q}}, \ldots\right\}$ denoted the set of abilities that is not yet available for the team to meet the task requirements.

Definition 2. (Team affordability) Given a set of robots in team $T M=\left\{r_{1}, r_{2}, \ldots r_{i}\right\}$, and bud is the budget of task, if

$$
f(\mathrm{~T}) \leq \text { bud }
$$

Then the team is said to be team affordability. Here $f(\mathrm{~T})$ is the total cost of robots to contribute abilities to task. i.e. $f(\mathrm{~T})=\sum_{i=1}^{l} \cos _{i}$.
Definition 3. (Team efficiency)Let $\mathrm{TF}=\{T M, T A, U S, T P\}$ be a team formation problem describe, if the robots in team has the abilities satisfied the requirements of task. i.e. $T A \supseteq \operatorname{Re}, U S=\varnothing$, and the team satisfied the team affordability, then the team is said to be team efficiency.

Definition 4. (Team stability) Let $\mathrm{TF}=\{T M, T A, U S, T P\}$ be a team formation problem describe, robot in team will not able to get more profit when is join another team. Then the team is said to be team stability.

Definition 5. (Team Robustness) Given a team $T F$, if any robot in team break down, the team can reach the goal as before. Then the team is said to be team robustness.

Definition 6. (Degree of Robust) Given a team $T F$ that satisfied team robustness, $n$ is the maximum number of robots that can leave the team, and the degree of robust of the team $D(T)=n$.

\section{Mechanism}

\subsection{Mechanism Design}

The mechanism select a coordinator at first, and analysis the requirement abilities of the task. According to the abilities of the task request, coordinator send offer to robots that satisfied the requirements. And Fig 1 is the structure diagram of the mechanism.

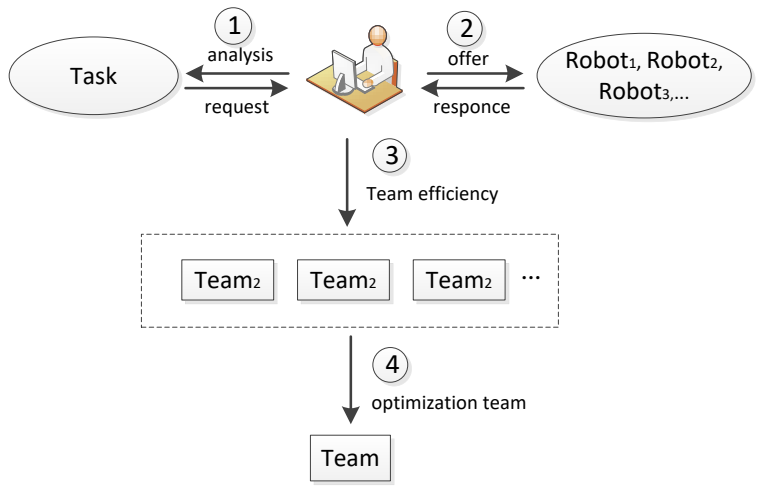

Fig 1. The structure diagram of the mechanism

\subsection{Coordinator Selection}

Task sponsor selected one coordinator among robots, The algorithm 1 is coordinator selection by the task sponsor.

Algorithm 1 CS
Input: Robots $=\left\{r_{1}, r_{2}, \ldots, r_{k}\right\}$
Output: Coordinator Co
// Count $_{i}$ is the number of ability that the Robot $r_{i}$ own.
// Cost $_{i}$ is the cost of Robot $r_{i}$ contribute all abilities
1. For $i=1 \rightarrow k$


2. $r_{i}, r_{j} \in$ Robots

3. If Count $_{i}>$ Count $_{j}$

4. $\quad \mathrm{Co} \leftarrow r_{i}$

5. Else if Count $_{i}=$ Count $_{j} \&$ Cost $_{i}<$ Cost $_{j}$

6. $\quad$ Co $\leftarrow r_{i}$

7. End if

8. End if

The algorithm realized the selection of the team coordinator from the set of candidate robots, The first step, all of the candidate robots in the selection of recycling for team coordinator, step two and three compare any robots in the candidate, and choose the team coordinator with the maximum count of abilities in step four, if there is no maximum count of abilities, then choose the minimum cost robot among the robots with maximum count of abilities as the team coordinator.

\subsection{Negotiation}

The coordinator matching robots to the requirement of the task, and negotiate to robot about whether to join the team, and coordinator choose the right robot to join the team at last.

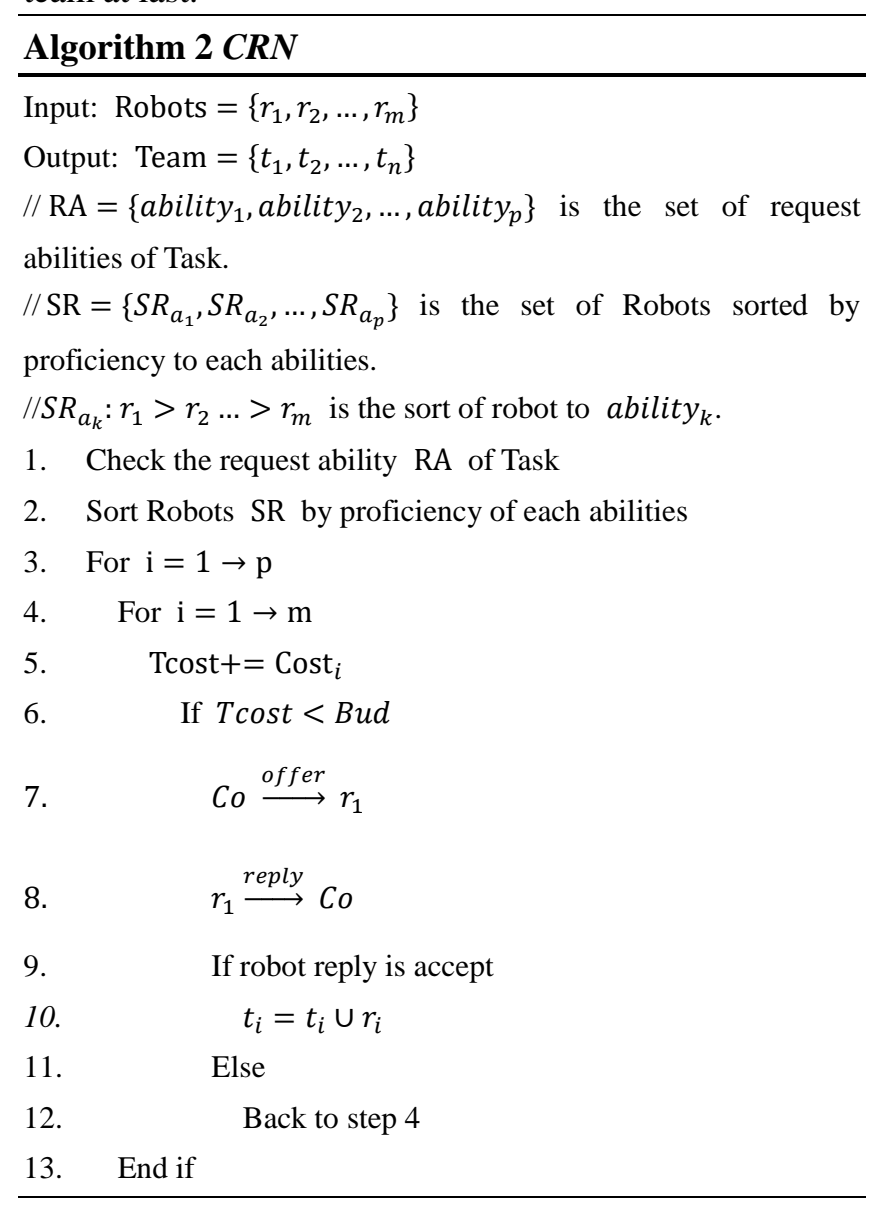

The algorithm realized the formation of robot team.
The first step, team coordinator do some task analysis, step two sorted the robots by the proficiency of abilities, and look for robots who own the ability in step three and four, in step five, calculate the total cost of the temporary team, and judge if it is smaller than the budget of the team in step six, the coordinator send offer to the robot and wait for the reply of robot in step seven and eight. If the robot replies accept to team coordinator, then make the robot join the team. If the robot replies refuse then back to step four and begin another round, until the requirements of the task are all satisfied in step twelve.

\subsection{Optimization Team}

By the algorithm 2, there are several team can be formatted to complete the task, in this paper, we Introducing the concept of robustness, and choose a team to complete the task by degree of robust.

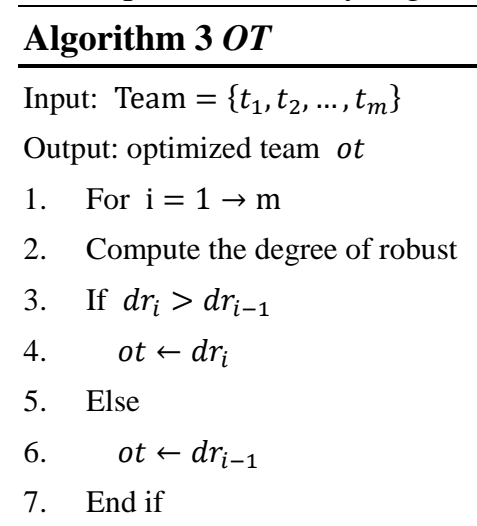

This algorithm selects an optimization team with the highest degree of robust from multiple teams. Cycled to the set of teams, and compared the team robustness, select a high degree of robustness of the team to complete the task finally.

\section{Example}

In this chapter, an application example is given to explain the working process of the mechanism. Task attributes describe Task $=\left\langle S p,\left(a_{1}, a_{2}, a_{4}, a_{6}, a_{7}\right), 50\right\rangle$, $\left\{a_{1}, a_{2}, a_{4}, a_{6}, a_{7}\right\}$ is the set of abilities that the task request, and 50 is the budget of task cost. The set of robots participate in perform the task denoted by Robot $=$ $\left\{\mathrm{R}_{1}, \mathrm{R}_{2}, \mathrm{R}_{3}, \mathrm{R}_{4}, \mathrm{R}_{5}, \mathrm{R}_{6}, \mathrm{R}_{7}\right\}$, and the attributes describe like Table 1, and proficiency is the proficiency of each ability.

Table 1

\begin{tabular}{c|c}
\hline Robot & $\langle$ ability, cost, proficiency $\rangle$ \\
\hline$R_{1}$ & $\left\langle a_{2}, 6,0.4\right\rangle,\left\langle a_{4}, 10,0.5\right\rangle$ \\
\hline
\end{tabular}




\begin{tabular}{c|c}
\hline$R_{2}$ & $\left\langle a_{1}, 7,0.6\right\rangle,\left\langle a_{3}, 9,0.7\right\rangle$ \\
\hline$R_{3}$ & $\left\langle a_{3}, 8,0.7\right\rangle,\left\langle a_{5}, 10,0.7\right\rangle,\left\langle a_{7}, 8,0.7\right\rangle$ \\
\hline$R_{4}$ & $\left\langle a_{1}, 8,0.6\right\rangle,\left\langle a_{2}, 7,0.5\right\rangle,\left\langle a_{6}, 15,0.6\right\rangle$ \\
\hline$R_{5}$ & $\left\langle a_{2}, 8,0.6\right\rangle,\left\langle a_{6}, 12,0.7\right\rangle$ \\
\hline$R_{6}$ & $\left\langle a_{4}, 16,0.9\right\rangle,\left\langle a_{6}, 18,0.8\right\rangle$ \\
\hline$R_{7}$ & $\left\langle a_{1}, 7,0.8\right\rangle,\left\langle a_{7}, 14,0.6\right\rangle$ \\
\hline
\end{tabular}

1) The selection of the team coordinator: Consider robots $R_{3}$ and $R_{4}$ have the same number of abilities, the total cost is 26 when robot $R_{3}$ contribution all abilities, less than 30 the total cost of robot $R_{4}$ when it contribution all abilities. So the task promoters select the robot $R_{3}$ as a team coordinator to complete the task.

2)Team formation: Take a rank of robots by the robot proficiency. Coordinator $\mathrm{R}_{3}$ send offer to robots, by the rank of robots. Consider the different reply of robot, there are 70 kinds of team and 20 of them own the property of team effectiveness and team robustness. By comparing the robustness of the teams, select three teams with the highest degree of robust. $f(\mathrm{~T})$ is the total cost of the team, and $D(T)$ is the degree of robust of the team, in Table 2.

Table 2

\begin{tabular}{c|c|l|l}
\hline Team & $T M$ & $f(\mathrm{~T})$ & $D(T)$ \\
\hline$t_{1}$ & $\left\{R_{7}, R_{4}, R_{1}, R_{5}, R_{3}\right\}$ & 44 & 2 \\
\hline$t_{2}$ & $\left\{R_{4}, R_{1}, R_{6}, R_{5}, R_{7}\right\}$ & 50 & 2 \\
\hline$t_{3}$ & $\left\{R_{2}, R_{1}, R_{6}, R_{5}, R_{7}\right\}$ & 48 & 2 \\
\hline
\end{tabular}

According to design of the mechanism, in the case of the same robustness, select the team with the smallest degree of robust as the optimization team to perform the task.Team $=\left\{R_{7}, R_{4}, R_{1}, R_{5}, R_{3}\right\}$ as the optimization team to complete the task.

\section{Conclusions}

One of the most important problems in artificial intelligence is how to realize the cooperation of multi robots, complex tasks can easily completed through the cooperation of multiple robots. Based on the problem of how to form a cooperative team, in this paper we proposed a robust mechanism for robot team formation. When team members can not contribute appointed skills to team, other robots can still complete the task instead, to ensure the effectiveness of the team. An example is given to illustrate the robustness of the mechanism. Compared with the existing robot team formation mechanism, RMTF increased the team's robustness, and ensured the stability of the team to complete the task.

\section{Acknowledgment}

Project supported by the National Nature Science Foundation of China (Grant No.61170201, No.61070133, No.61472344); Six talent peaks project in Jiangsu Province(Grant No.2011-DZXX-032). Innovation Foundation for graduate students of Jiangsu Province(Grant No.CXLX12 0916), Jiangsu Science and Technology Project No. BY2015061-06、BY2015061-08, Yangzhou Science and Technology Project No. SXT20140048, SXT20150014, SXT201510013, Natural Science Foundation of the Jiangsu Higher Education Institutions (Grant No.14KJB520041), Innovation Program for graduate students of Jiangsu Province(Grant No.SJZZ16_0261).

\section{References}

(1) Wang W, Jiang Y. A Practical Negotiation-Based Team Formation Model for Non-cooperative Social Networks[J]. 2014:344-351.

(2) Nair R, Tambe M. Hybrid BDI-POMDP Framework for Multiagent Teaming[J]. Computer Science, 2011, 23:2005.

(3) Marcolino L S, Jiang A X, Tambe M. Multi-agent team formation: diversity beats strength?[C]// International Joint Conference on Artificial Intelligence. 2013:279-285.

(4) Bachrach Y, Rosenschein J S. Coalitional skill games[C]// International Joint Conference on Autonomous Agents and Multiagent Systems. 2008:1023-1030.

(5) Okimoto T, Schwind N, Clément M, et al. How to Form a Task-Oriented Robust Team[C]// The, International Conference on Autonomous Agents and Multiagent Systems. 2015.

(6) Vidal, Jos\&\#. The effects of cooperation on multiagent search in task-oriented domains[C]// International Joint Conference on Autonomous Agents \& Multiagent Systems. ACM, 2002:453-454.

(7) Dasgupta P, Cheng K. Robust Multi-robot Team Formations Using Weighted Voting Games[M]// Distributed Autonomous Robotic Systems. Springer Berlin Heidelberg, 2012:373-387.

(8) Kaminka G A, Frenkel I. Towards flexible teamwork in behavior-based robots: extended abstract[C]// International Joint Conference on Autonomous Agents and Multiagent Systems. 2005:1355-1356.

(9) Liemhetcharat S, Veloso M. Forming an effective multi-robot team robust to failures[C]// Ieee/rsj International Conference on Intelligent Robots and Systems. IEEE, 2013:5240-5245. 